\title{
PROFINITE ORTHOMODULAR LATTICES
}

\author{
TAE HO CHOE AND RICHARD J. GREECHIE
}

(Communicated by Jeffry N. Kahn)

\begin{abstract}
We prove that any compact topological orthomodular lattice $L$ is zero dimensional. This leads one to show that $L$ is profinite iff it is the product of finite orthomodular lattices with their discrete topologies. We construct a completion $\bar{L}$ of a residually finite orthomodular lattice $L$ having the property that every element of $\bar{L}$ is the join of meets of elements of $L$. Necessary and sufficient conditions for $L$ that $\bar{L}$ is the MacNeille completion are obtained.
\end{abstract}

In this paper, we initiate the study of topological orthomodular lattices (abbreviated: TOMLs). We prove that any compact TOML is zero dimensional and atomic; and, further, we provide a simple description of such a compact topology in terms of the intervals generated by atoms and coatoms. This leads to determining that the profinite OMLs are precisely the products of finite orthomodular lattices with their discrete topologies.

We introduce a zero-dimensional completion $\bar{L}$ of a residually finite OML $L$. $\bar{L}$ is a completion of $L$ having the property that every element of $\bar{L}$ is the join of meets of elements of (an isomorphic copy of) $L$. This result is of interest, because the MacNeille completion of an OML need not be again an OML; thus there is an OML $P$ such that there is no completion of $P$ with the property that each of its elements is the join of elements of $P$. Essentially the only known example of this phenomenon is the OML of all finite or cofinitedimensional subspaces of an inner product space (over the reals or complexes) which is not a Hilbert space [4]. Finally, we show that $\bar{L}$ is the MacNeille completion of $L$ if and only if the center of $\bar{L}$ is isomorphic to the MacNeille completion of the center of $L$.

By a topological lattice, we mean a pair $(L, \tau)$, where $L$ is a lattice and $\tau$ is a Hausdorff topology on $L$ for which the two lattice-operations are continuous.

For a lattice $(L, \wedge, \vee)$ and for a pair of subsets $A$ and $B$ of $L$, we shall use $A \wedge B$ and $A \vee B$ to denote the set $\{a \wedge b \mid a \in A$ and $b \in B\}$ and $\{a \vee b \mid a \in A$ and $b \in B\}$, respectively. It is known [5] that if $L$ is a topological lattice and if $A$ is a compact subset of $L$, then $A \wedge L$ and $A \vee L$ are both closed, and hence $a \wedge L$ and $a \vee L$ are both closed for each $a \in L$.

The following facts are well known in the theory of topological lattices:

Received by the editors November 28, 1988 and, in revised form, January 3, 1992.

1991 Mathematics Subject Classification. Primary 06F30.

The first author's research was supported by the NSERC. 
Fact $1[5,12]$. Every compact lattice is a complete lattice.

Fact 2 [5]. The connected component of an element in a topological lattice is a closed convex sublattice.

Fact 3 [1]. If $U$ is open in a topological lattice then so are $U \wedge L$ and $U \vee L$.

Fact $4[8,12]$. Every compact Boolean algebra is complete and atomic, i.e., it is isomorphic to a power set lattice.

A topological orthomodular lattice is defined to be an OML, which is a topological lattice for which the orthocomplementation operation is also continuous.

For the other terminologies and definitions we mainly follow those in [9] for OMLs, and those in $[5,12]$ for topological lattices.

\section{Topological OMLs}

In this section we observe basic properties of TOMLs and prove that a compact TOML must be totally disconnected (zero-dimensional) and atomic; moreover, its topology is completely characterized by the atoms of $L$. For a subset $M$ of a TOML, $M^{\prime}$ is the set $\left\{m^{\prime} \mid m \in M\right\}$.

Lemma 1. Let $L$ be a TOML. If there exists an isolated point in $L$, then $L$ is a discrete TOML.

Proof. First, we prove the lemma in the case that 0 is an isolated point. Take any element $x \in L$. Since $x \wedge x^{\prime}=0$, there exists neighborhoods (=nbds) $U_{1}$ and $U_{2}$ of $x$ such that $U_{1} \wedge U_{2}^{\prime}=\{0\}$. Setting $U_{1} \cap U_{2}=W_{1}$, we have $W_{1} \wedge W_{1}^{\prime}=\{0\}$. There are nbds $U_{3}$ and $U_{4}$ of $x$ such that $U_{3} \wedge U_{3} \subset W_{1}$ and $U_{4} \vee U_{4} \subset W_{1}$ since $x \wedge x=x$ and $x \vee x=x$. Setting $W_{2}=U_{3} \cap U_{4}$, we have $W_{2} \wedge W_{2} \subset W_{1}$ and $W_{2} \vee W_{2} \subset W_{1}$. We claim that $W_{1} \cap W_{2}=\{x\}$. Indeed, if $y \in W_{1} \cap W_{2}$, then $x \vee y=(x \wedge y) \vee\left[(x \vee y) \wedge(x \wedge y)^{\prime}\right]$. Since $x \vee y$ and $x \wedge y \in W_{1}$, we have $(x \vee y) \wedge(x \wedge y)^{\prime}=0$, and hence $x=y$. Thus $L$ is discrete. Now if $x$ is any isolated point in $L$, then there exist nbds $U$ and $U_{0}$ of $x$ and 0 , respectively, such that $U \vee U_{0}=\{x\}$. It follows that $U_{0} \subset x \wedge L$. On the other hand, $x \wedge L$ is itself a TOML under its relative topology. Therefore, the unit element $x$ in $x \wedge L$ is an isolated point. Hence the (relative) orthocomplement 0 in $x \wedge L$ of $x$ is an isolated point. Thus there exists a nbd $V$ of 0 in $L$ such that $V \cap(x \wedge L)=\{0\}$. Hence $V \cap U_{0}=\{0\}$. The proof is complete.

Recall that a block of an OML $L$ is a Boolean subalgebra maximal with respect to set theoretic inclusion. It is easy to see that the blocks of $L$ are precisely the subsets $B$ of $L$ satisfying $B=C(B)$ where $C(B)$ is the commutator of $B$.

Lemma 2. If $L$ is a TOML, then for each $a \in L$, the commutator $C(a)$ of a is closed, and hence every block is closed.

Proof. If $\left\{x_{\alpha} \mid \alpha \in D\right\}$ is a net in $C(a)$, which converges to $x$, then the net $\left\{\left(x_{\alpha} \vee a\right) \wedge\left(x_{\alpha} \vee a^{\prime}\right) \mid \alpha \in D\right\}$, which is the same as $\left\{x_{\alpha}\right\}$, converges to $(x \vee a) \wedge\left(x \vee a^{\prime}\right)$, which is $x$. Thus $x \in C(a)$. If $B$ is a block of $L$, then $B=C(B)=\bigcap\{C(b) \mid b \in B\}$ is closed.

Corollary 1. If $L$ is a compact TOML, then

(i) the center $C(L)$ of $L$ is isomorphic to a power set lattice, and

(ii) every block is also isomorphic to a power set lattice. 
Proof. This follows from Lemma 2 and Fact 4.

Corollary 2. If $L$ is a compact TOML, then $L$ is atomic.

Proof. It is easy to see that every atom of a block is also an atom of $L$. Hence every nonzero element of $L$ dominates an atom of $L$, since it is in some block.

Lemma 3. Every compact TOML is totally disconnected.

Proof. Let $C$ be the connected component of an element $x$ of $L$. By Fact $2, C$ is a closed convex sublattice of $L$. Thus $C$ is a compact topological lattice with its relative topology. By Fact $1, C=[z, y](=z \vee(y \wedge L)$ where $z \leq y)$ for some $z$ and for $y \in L$. Assume that $z<y$. Then $y \wedge z^{\prime}>0$. By Corollary 2 there exists an atom $a$ of $L$ such that $0<a \leq y \wedge z^{\prime}$. Then $a \wedge C$ is connected, since it is the image of $C$ under a continuous map. On the other hand, $a \wedge C=[0, a]=\{0, a\}$, which is not connected. This is a contradiction. Hence $z=y=x$ and $C=\{x\}$.

Lemma 4. If $L$ is a TOML, then for any atom a of $L, a \vee L$ is open, and hence $a^{\prime} \wedge L$ is open.

Proof. Take a nbd $U$ of $a$ with $0 \notin U$, and take an open nbd $V$ of $a$ such that $V \wedge V \subset U$. Then we have $V \subset a \vee L$ because $0 \notin U$. It follows that $a \vee L=V \vee L$, which is open by Fact 3.

Theorem 1. The topology $\tau$ of a compact TOML $L$ is generated by the family $\left\{a \vee L, a^{\prime} \wedge L \mid a\right.$ is an atom of $\left.L\right\}$.

Proof. Let $\mu$ be the topology on $L$ generated by the family of the intervals $a \vee L$ and $a^{\prime} \wedge L$ for all atoms $a$ of $L$. By Lemma 4, $\mu \leq \tau$. We now show that $\mu$ is Hausdorff. For $x \not \leq y$, there exists an atom $a$ of $L$ such that $a \leq x$ and $a \leq y$. Therefore, since $y^{\prime} \leq a^{\prime}$, again we have an atom $b$ of $L$ such that $y \leq b^{\prime}$ and $a \not b^{\prime}$. Clearly $(a \vee L) \cap\left(b^{\prime} \wedge L\right)=\varnothing$, where $a \vee L$ and $b^{\prime} \wedge L$ are both open subsets containing $x$ and $y$, respectively, by Lemma 4. Consider the identity map $i:(L, \tau) \rightarrow(L, \mu)$. Then $i$ is continuous because $\mu \leq \tau$. Furthermore, $i$ is a closed map because $\tau$ is compact and $\mu$ is Hausdorff. Recall that a closed continuous bijection is a homeomorphism. Hence we have $\tau=\mu$.

\section{Profinite OMLs}

By a profinite $O M L$ we mean a compact TOML $L$, which is a projective limit (i.e., inverse limit) of finite OMLs with their discrete topologies; an equivalent condition $[2,8]$ is that the intersection of all closed and open (= clopen) congruences on $L$ is trivial. The following question has interested many authors [2, 8] and has still remained as open: what are necessary and sufficient conditions for a zero-dimensional compact universal algebra to be profinite. Among many different partial solutions of the question, it is proved [6] that if the universal algebra is (generalized) associative and distributive, then every zero-dimensional compact universal algebra is profinite. Typical examples of profinite algebras of any type are all discrete finite algebras or, more generally, any product of finite algebras with their discrete topologies. In this section, we characterize profinite OMLs as follows: A compact OML is profinite iff it is a product of finite OMLs with their discrete topologies. However, the above question in the case of OML 
is still open. Namely, is every compact TOML (and hence zero dimensional by Lemma 3) a product of finite TOMLs?

Recall that any compact TOML is zero dimensional and atomic; furthermore, the center $C(L)$ is always closed for any TOML $L$. Therefore, in the compact case, $C(L)$ is itself a complete atomic Boolean algebra.

Lemma 5. Let $L$ be a compact TOML. If $a \in C(L)$ and if $a \wedge L$ is open in $L$, then $a=q_{1} \wedge \cdots \wedge q_{n}$ for finitely many coatoms $q_{k}$ of $C(L)$, and furthermore, $q_{k} \wedge L$ is also open for $k=1,2, \ldots, n$.

Proof. Since $\left(C(L), \tau_{c}\right)$ is a compact Boolean algebra under its relative topology $\tau_{c}$, it is algebraically and topologically isomorphic to the power set lattice $\left(2^{|A|}, \tau_{p}\right)$, where $A$ is the set of atoms of $C(L)$ and $\tau_{p}$ is the product topology of two point discrete spaces. Let $\Downarrow a=(a \wedge L) \cap C(L) \quad(=a \wedge C(L))$. Then $\Downarrow a$ is an open subset in $C(L)$ (or in $2^{|A|}$ ). Since the product topology $\tau_{p}$ has a subbasis $\left\{\Downarrow q_{\alpha}, \Uparrow p_{\beta} \mid q_{\alpha}^{\prime} \in A, p_{\beta} \in A\right\}, \Downarrow a$ is a union of intervals of the form $\left[p_{1} \wedge \cdots \wedge p_{m}, q_{1} \wedge \cdots \wedge q_{n}\right]$ in $C(L)$ (or in $2^{|A|}$ ) where $p_{j}$ and $q_{k}^{\prime} \in A: j=1,2, \ldots, m$ and $k=1,2, \ldots, n$. Hence $a=q_{1} \wedge \cdots \wedge q_{n}$ for finite coatoms $q_{k}$ of $C(L)$. Now show that $q_{k} \wedge L$ is also open in $L$ for $k=1,2, \ldots, n$. Assume that $q_{1} \wedge L$ is not open in $L$. Then there exists a net $\left\{x_{\alpha} \mid \alpha \in D\right\}$ in $L-\left(q_{1} \wedge L\right)$ such that it converges to $x$ and $x \in q_{1} \wedge L$. Clearly $\left\{x_{\alpha} \wedge q_{2} \wedge \cdots \wedge q_{n}\right\}$ converges to $x \wedge q_{2} \wedge \cdots \wedge q_{n}$. On the other hand, $x_{\alpha} \wedge q_{2} \wedge \cdots \wedge q_{n} \notin a \wedge L$ for any $\alpha \in D$, but $x \wedge q_{2} \wedge \cdots \wedge q_{n} \in a \wedge L$, which is open by hypothesis. This is a contradiction. Similarly, $q_{k} \wedge L$ is open for $k=2, \ldots, n$.

The following lemma is important in the sequel:

Lemma 6. If $L$ is a compact TOML, then $L$ is algebraically and topologically isomorphic to the product $\prod_{p \in A}(p \wedge L)$, where $A$ is the set of atoms of $C(L)$ and each $p \wedge L$ has its relative topology.

Proof. It is well known that the map $\delta: L \rightarrow \prod_{p \in A}(p \wedge L)$ defined by $\delta(x)=$ $(p \wedge x)_{p \in A}$ is an algebraical isomorphism. Since $\Pi(p \wedge L)$ has the product topology, $\delta$ is continuous if and only if $q_{p} \circ \delta$ is continuous for any $p \in A$, where $q_{p}: \Pi(p \wedge L) \rightarrow p \wedge L$ is the $p$ th projection. But $\left(q_{p} \circ \delta\right)(x)=p \wedge x$ for each $x \in L$, which is clearly continuous for each $p \in A$. Furthermore, $L$ is compact and $\Pi(p \wedge L)$ is Hausdorff. It follows that $\delta$ is a homeomorphism.

We are now in a position to prove our main theorem.

Theorem 2. Let $L$ be a compact TOML. Then the following statements are equivalent:

(i) $L$ is a profinite $O M L$.

(ii) For any $x \neq 0$, there exists $a \in C(L)$ such that $a \wedge L$ is open in $L$ and $x \notin a$.

(iii) For any coatom $q$ of $C(L), q \wedge L$ is open in $L$.

(iv) $L$ is algebraically and topologically isomorphic to a product of finite OMLs with their discrete topologies.

Proof. (i) $\Rightarrow$ (ii). Since the intersection $\bigcap\left\{\theta_{i} \mid i \in I\right\}$ of clopen congruences on $L$ is the trivial congruence $\Delta_{L}$, for any $x \neq 0$ there exists $\theta_{i}$ such that $x \notin 0 / \theta_{i} \quad(=$ the congruence class containing 0$)$. Note that $0 / \theta_{i}$ is a clopen 
$p$-ideal of $L$. Thus $0 / \theta_{i}$ is a convex compact sublattice of $L$, and hence it is of the form $a \wedge L$ for some $a \in L$. Therefore $a \in C(L)$ and $x \leq \leq a$, because $\left(a \vee y^{\prime}\right) \wedge y$ is perspective to $\left(y \vee a^{\prime}\right) \wedge a$ for all $y \in L$ by the parallelogram law.

(ii) $\Rightarrow$ (iii). For $x \neq 0$, there exists $a \in C(L)$ such that $a \wedge L$ is open and $x \not a$. By Lemma 5, $a=q_{1} \wedge \cdots \wedge q_{n}$ for coatoms $q_{k}$ of $C(L)$ and $q_{k} \wedge L$ is open for $k=1,2, \ldots, n$. Thus $x \not \leq q_{k}$ for some $k$. Hence for any $x \neq 0$, there exists a coatom $q_{0}$, say, such that $x \not \leq q_{0}$ and $q_{0} \wedge L$ is open. Now let $q$ be any coatom of $C(L)$. We claim that $q \wedge L$ is open in $L$. Indeed, letting $x=q^{\prime}$ in the above, we have a coatom $q_{0}$ of $C(L)$ such that $q^{\prime} \not q_{0}$ and $q_{0} \wedge L$ is open. But $q_{0}$ must be $q$ since $C(L)$ is a Boolean algebra, proving that $q \wedge L$ is open in $L$.

(iii) $\Rightarrow$ (iv). Let $\left\{a_{i} \mid i \in I\right\}$ be the set of all atoms of $L$, and let $A$ be the set of all atoms of $C(L)$. Now take any coatom $q$ of $C(L)$. By Corollary 2, Lemma 4, and (iii), $\left\{a_{i} \vee L \mid i \in I\right.$ and $\left.a_{i} \not \leq q\right\} \cup\{q \wedge L\}$ is an open cover of $L$. Therefore, there exists a finite subcover $\left\{a_{k} \vee L \mid k=1,2, \ldots, n\right\} \cup\{q \wedge L\}$. It follows that $q^{\prime}=\sup \left\{a_{k} \mid k=1,2, \ldots, n\right\}$ and $q^{\prime} \in A$. Thus $q^{\prime} \wedge L$ is a finite OML for each coatom $q$ of $C(L)$. By Lemma 6 , we have $L \cong \prod_{p \in A}(p \wedge L)$, where $p \wedge L$ has its discrete topology for each $p \in A$.

(iv) $\Rightarrow(\mathrm{i})$. This is obvious.

Corollary 3. (i) Every irreducible profinite $O M L$ is finite.

(ii) Let $L$ be a compact TOML. Then $L$ is profinite iff each atom of $p$ of $C(L)$ is a finite join of atoms of $L$.

(iii) Let $L$ be a compact TOML with finitely many blocks. Then $L$ is profinite iff $L$ is isomorphic to $B \times L_{0}$, where $B$ is a compact Boolean algebra and $L_{0}$ is a finite discrete $O M L$.

Proof. (i) and (ii) are obvious. For (iii), by Theorem (1.2) of [3], $L \cong B \times$ $L_{1} \times \cdots \times L_{n}$ algebraically, where $B$ is a Boolean algebra and each $L_{i}$ is an irreducible OML. Considering the corresponding relative topologies for each of the factors $B, L_{1}, \ldots, L_{n}$, we have that the isomorphism is topological as well because $L$ is compact and the isomorphism is continuous. Clearly, each $L_{i}$ with its relative topology is profinite because $L$ is. By (i), $L_{i}$ is finite $(i=1,2, \ldots, n)$. Setting $L_{0}=L_{1} \times \cdots \times L_{n}$, we have the required result. The converse is trivial.

\section{ZERO-DIMENSIONAL COMPLETIONS}

By a residually finite OML we mean an OML that can be algebraically embedded into a product of finite OMLs. The study that an OML can be embedded into a complete OML is an important work with implications in the axiomatic foundations of quantum mechanics (see [11]). For example, [4] studies the problem of finding a completion of a given OML, by a purely algebraic method. In this section, we define a special type of completion, called a zero-dimensional completion, characterize OMLs having such a completion-they turn out to be precisely the residually finite OMLs-and investigate conditions for an OML $L$ so that the zero-dimensional completion of $L$ is isomorphic to the MacNeille completion of $L$.

For an OML $L$, a profinite OML $\bar{L}$ is called a zero-dimensional completion of $L$ if there exists an (OML)-embedding $j: L \rightarrow \bar{L}$ such that $j(L)$ is a topologically dense subset of $\bar{L}$ (i.e., $j$ is a dense embedding). 
Theorem 3. Let $L$ be an $O M L . L$ is residually finite iff there exists a zerodimensional completion $\bar{L}$ of $L$ such that $p \wedge \bar{L}$ is a finite $O M L$ for each atom of the center of $\bar{L}$.

Proof. Suppose that $L$ is residually finite, and suppose that it is embedded into a product $\prod L_{i} \quad(i \in I)$ of finite OMLs. Let each $L_{i}$ have the discrete topology so that $\Pi L_{i}$ is a compact TOML. Setting $\bar{L}=\Gamma(j(L))$, where $\Gamma$ is the topological closure operation and $j$ is the embedding, we have a zerodimensional completion $\bar{L}$ of $L$ because the closure of a subalgebra of $\prod L_{i}$ is again a (compact) subalgebra. Hence $\bar{L}$ is profinite [2]. Let $A$ be the set of all atoms of $C(\bar{L})$. Now show that $p \wedge \bar{L}$ is a finite OML for each $p \in A$. Take $p \in A$, and let $q=p^{\prime}$. Then $q \wedge \bar{L}$ is clopen in $\bar{L}$ by Theorem 2 . Therefore, $q \wedge \bar{L}$ induces a clopen congruence $\theta_{q}$ on $\bar{L}$ such that $\bar{L} / \theta_{q} \cong p \wedge \bar{L}$, which is finite. Conversely, suppose that $\bar{L}$ is a zero-dimensional completion of $L$ such that $p \wedge \bar{L}$ is finite for any $p \in A$. By Theorem $2, \bar{L}$ is a product of finite OMLs $p \wedge \bar{L}$.

Henceforth, $A$ is the set of all atoms of $C(\bar{L})$ where $\bar{L}$ is a zero-dimensional completion of $L$.

Lemma 7. If $\bar{L}$ is a zero-dimensional completion of a residually finite $O M L L$ then, for each $p \in A, p$ is a meet of elements of $L$ (identifying $L$ and $J(L)$ ). Proof. Let $p \in A$ and let $q=p^{\prime}$. Then there exists a net $\left\{x_{\alpha} \mid \alpha \in D\right\}$ in $L$ ( $\equiv j(L))$ converging to $q$ in $\bar{L}$. Since $q \wedge \bar{L}$ is open, there exists $\alpha_{0} \in D$ such that $x_{\alpha} \in q \wedge L$ for all $\alpha \geq \alpha_{0}$. So we may assume that $\left\{x_{\alpha} \mid \alpha \in D\right\}$ is a net in $q \wedge L$. Now take any $r \in A$. Then the net $\left\{r \wedge x_{\alpha} \mid \alpha \in D\right\}$ converges to $r \wedge q$ in $r \wedge \bar{L}$, which is a finite OML. Thus there exists $\alpha_{1} \in D$ such that $r \wedge x_{\alpha}=r \wedge q$ for all $\alpha \geq \alpha_{1}$. It follows that

$$
\sup \left\{r \wedge x_{\alpha} \mid \alpha \geq \alpha_{1}\right\}=r \wedge \sup \left\{x_{\alpha} \mid \alpha \geq \alpha_{1}\right\}=r \wedge q \text { for each } r \in A .
$$

This implies that $\sup \left\{x_{\alpha} \mid \alpha \geq \alpha_{1}\right\}=q$. Hence $p=\inf \left\{x_{\alpha}^{\prime} \mid \alpha \geq \alpha_{1}\right\}$.

Lemma 8. Under the same hypothesis of Lemma 7, we have $p \wedge \bar{L}=p \wedge L$ in $\bar{L}$ for each $p \in A$.

Proof. Clearly $p \wedge L \subset p \wedge \bar{L}$ for each $p \in A$. If $p \wedge x \in p \wedge \bar{L}(x \in \bar{L})$, then $x_{\alpha} \rightarrow x$ for some net $\left\{x_{\alpha}\right\}$ in $L$. For any $p \in A, p \wedge x_{\alpha} \rightarrow p \wedge x$ in $p \wedge \bar{L}$. Hence, there exists an $\alpha_{0}$ such that $p \wedge x=p \wedge x_{\alpha}$ for all $\alpha \geq \alpha_{0}$. Thus $p \wedge x \in p \wedge L$.

Theorem 4. If $\bar{L}$ is a zero-dimensional completion of residually finite $O M L L$, then every element of $\bar{L}$ is a join of meets of elements of $L$ (identifying $L$ and $j(L))$.

Proof. Let $\delta$ be the isomorphism between $\bar{L}$ and $\prod_{p \in A}(p \wedge \bar{L})$. By Lemma 8, $\prod_{p \in A}(p \wedge \bar{L})=\prod_{p \in A}(p \wedge L)$. Let $x \in \bar{L}$. Then for each $p \in A$ there exists $x_{p} \in L$ such that $\delta(x)=(p \wedge x)_{p \in A}=\left(p \wedge x_{p}\right)_{p \in A}$. By Lemma 7, we have for each $p$ in $A, p=\inf y_{\alpha, p}$ where $y_{\alpha, p} \in L$. Therefore

$$
x=\sup _{p \in A}\left(p \wedge x_{p}\right)=\sup \left[\inf \left(y_{\alpha, p}\right) \wedge x_{p}\right] .
$$


Remark. The above result shows that any residually finite OML $L$ has a completion, each element of which is a join of meets of elements of $L$. Such a completion was first obtained (strictly by algebraic means) by Bruns and Greechie (unpublished).

Let $L$ be a poset. A complete lattice $\widetilde{L}$ in which $L$ is embedded is called a MacNeille completion of $L$ if $L$ is join dense and meet dense in $\widetilde{L}$. Any two MacNeille completions of a poset are isomorphic. A MacNeille completion of an ortholattice $L$ inherits, in a natural way, an orthocomplementation which extends that of $L$. We thus regard a MacNeille completion of an ortholattice as an ortholattice.

Theorem 5. Let $L$ be a residually finite $O M L$ and $\bar{L}$ a zero-dimensional completion of $L$. Then the following statements are equivalent:

(i) $\bar{L}$ is isomorphic to $\tilde{L}$.

(ii) Every atom of the center of $\bar{L}$ belongs to $L$ (identifying $L$ with $j(L)$ ).

(iii) $C(\bar{L})$ is isomorphic to the MacNeille completion of $C(L)$.

Proof. (i) $\Rightarrow$ (ii). Let $A$ be the set of all atoms of $C(\bar{L})$ again. Take $p \in A$. Since $\bar{L} \cong \tilde{L}, p=\sup \left\{x_{\alpha}\right\}$ for some $\left\{x_{\alpha}\right\} \subset L$. Thus $x_{\alpha} \in p \wedge \bar{L}$ for all $\alpha$. But $p \wedge \bar{L}$ is a finite OML. Therefore, the sup must be a finite sup, and hence $p \in L$.

(ii) $\Rightarrow$ (i). Again let $\delta$ be the isomorphism between $\bar{L}$ and $\prod_{p \in A}(p \wedge \bar{L})$. For any $x \in \bar{L}, \delta(x)=(p \wedge x)_{p \in A}=\left(p \wedge x_{p}\right)_{p \in A}$ where $p \wedge x_{p} \in L$ for each $p \in A$. Since $\delta\left(p \wedge x_{p}\right)=\left(\ldots, 0, \ldots, 0, p \wedge x_{p}, 0, \ldots\right) \in \delta(L)$, we have $\delta(x)=\sup _{p \in A} \delta\left(p \wedge x_{p}\right)$. Hence $\delta(L)$ (equivalently $L$ ) is join dense in $\delta(\bar{L})$ (equivalently $\bar{L}$ ). Dually $\delta(L)$ is also meet dense in $\delta(\bar{L})$.

(ii) $\Rightarrow$ (iii). We claim that $C(L) \subset C(\bar{L})$. Indeed, if $c \in C(L)$ and $z \in \bar{L}$ then $x_{\alpha} \rightarrow z$ for some net $\left\{x_{\alpha}\right\}$ in $L$. Since $c$ commutes with every $x_{\alpha}, c$ commutes with $z$. Since $A \subset C(L)$ and every element of $C(\bar{L})$ is a join of elements of $A, C(L)$ is join dense and hence meet dense in $C(\bar{L})$.

(iii) $\Rightarrow$ (ii). For each $p \in A, p=\sup \left\{b_{\alpha}\right\}$ for some $\left\{b_{\alpha}\right\} \subseteq C(L)$. Since $C(L) \subset C(\bar{L}), b_{\alpha} \in\{0, p\}$ for all $\alpha$. Hence $p=b_{\alpha}$ for some $\alpha$. The proof is complete.

\section{REFERENCES}

1. L. W. Anderson, One dimension topological lattices, Proc. Amer. Math. Soc. 10 (1959), 715-720.

2. B. Banaschewski, On profinite universal algebras, Proc. Third Topological Conf., Prague, September 1971.

3. G. Bruns, Block-finite orthomodular lattices, Canad. J. Math. 31 (1979), 961-985.

4. G. Bruns, R. J. Greechie, J. Harding, and M. Roddy, Completions of orthomodular lattices (to appear).

5. T. H. Choe, On compact topological lattices of finite dimension, Trans. Amer. Math. Soc. 40 (1969), 223-237.

6. Z Z Z Z _ dimensional compact associative distributive universal algebras, Proc. Amer. Math. Soc. 42 (1974), 607-613.

7. R. J. Greechie, On generating distributive sublattices of orthomodular lattices, Proc. Amer. Math. Soc. 67 (1977), 17-22.

8. P. T. Johnstone, Stone spaces, Cambridge Univ. Press, London and New York, 1982. 
9. G. Kalmback, Orthomodular lattices, Academic Press, New York, 1982.

10. J. D. Lawson, Intrinsic topologies in topological lattices and semilattices, Pacific J. Math. 44 (1973), 593-602.

11. G. W. Mackey, Mathematical foundation of quantum mechanics, Benjamin, New York, 1963.

12. D. P. Strauss, Topological lattices, Proc. London Math. Soc. (3) 18 (1968), 217-230.

Department of Mathematics, McMaster University, Hamilton, Ontario, Canada L8S 4K1

Department of Mathematics, Kansas State University, Manhattan, Kansas 66506 